ORIGINAL RESEARCH

\title{
Efficacy of Topical Treatments for Chrysaora chinensis Species: A Human Model in Comparison with an In Vitro Model
}

\author{
Matthieu P. DeClerck, MD; Yvonne Bailey, MD; David Craig, MD; Michelle Lin, BA; Lauren J. Auerbach, BS; \\ Olivia Linney, BS; Doug E. Morrison, MS; Wyatt Patry, BS; Paul S. Auerbach, MD, MS \\ From the Division of Emergency Medicine, Department of Surgery, Stanford University School of Medicine, Stanford, CA (Drs CeClerck, Bailey, \\ Craig, and P. Auerbach; Ms Lin; and Ms L. Auerbach); the Oregon Health and Science University School of Medicine, Portland, OR (Ms Linney); \\ the Department of Surgery, Stanford University School of Medicine, Stanford, CA (Mr Morrison); and the Division of Husbandry, Monterey Bay \\ Aquarium, Monterey, CA (Mr Patry).
}

\begin{abstract}
Objectives. - This study sought to create a model for testing topical treatment of jellyfish stings. It sought to determine which treatments 1) stimulate/inhibit nematocyst discharge; 2) decrease pain; and 3) decrease skin inflammation; it also sought to discover whether there is a clinical correlation between stimulated nematocyst discharge observed in vitro to the pain and erythema experienced by humans stung by a particular species of jellyfish, $C$ chinensis.

Methods.-Chrysaora chinensis stung 96 human subjects, who were then treated with isopropyl alcohol, hot water, acetic acid, papain meat tenderizer, lidocaine, or sodium bicarbonate. Pain and erythema were measured. In a separate experiment, nematocysts were examined microscopically after exposure to the same topical treatments used in the human experiment.

Results.-Forearms treated with papain showed decreased mean pain over the first 30 minutes after being stung, relative to placebo, although only by a small amount. The other topical treatments tested did not reach statistical significance. Sodium bicarbonate may reduce erythema after 30 minutes of treatment; sodium bicarbonate and papain may reduce erythema at 60 minutes. The other topical treatments tested did not reach statistical significance. Nematocyst discharge in vitro occurred when tentacles of $C$ chinensis were exposed to acetic acid or isopropyl alcohol. Sodium bicarbonate, papain, heated water, and lidocaine did not induce nematocyst discharge.

Conclusions.-Papain-containing meat tenderizer used as a topical treatment for $C$ chinensis stings may decrease pain. Although there is published experimental support for the concept that in vitro nematocyst discharge correlates with in vivo human pain perception, no definitive randomized controlled trial, including ours, has yet provided incontrovertible evidence of this assertion. Despite this study's limitations, it presents a viable basis for future human studies looking at the efficacy of topical treatments for jellyfish stings.
\end{abstract}

\section{Introduction}

Jellyfish stings upon humans are worldwide occurrences, with an estimated 150 million people stung annually. ${ }^{1}$ Jellyfish of the phylum Cnidaria envenom prey by using stinging organelles (nematocysts) that are contained within nematocytes lining the tentacles and mouth. Nematocysts comprise a secretory organelle (cnida) with a capsule containing a coiled, barb-laden tubule

Corresponding author: Matthieu P. DeClerck, MD, 743 S. Mentor Ave, Pasadena, CA 91106 (e-mail: mpdeclerck@gmail.com). (nema), trigger (cnidocil), and venom. Envenomation occurs upon mechanical or chemical stimulation of the cnidocil, which leads to injection of the nema into the victim and venom release. $^{2}$ A single jellyfish may carry millions of nematocysts. An individual nematocyst can discharge its nema with a velocity of $2 \mathrm{~m} / \mathrm{s}$, corresponding to an acceleration of $40,000 \mathrm{~g}$, ensuring effective penetration of human skin and envenomation. $^{2,3}$

There exist thousands of jellyfish species, many of which are dangerous to humans. ${ }^{3}$ The nematocysts and venom of the majority of these species have not been not 
well characterized, but it is clear that variability exists in nematocyst structure and venom composition. There are at least 30 morphological types of nematocysts. ${ }^{4}$ Similarly, clinical manifestations of jellyfish stings vary widely. Reactions range from minor local skin eruption with pain (most common) to systemic toxicity and death. Many jellyfish venoms contain a dermonecrotic component, which causes local skin inflammation and necrosis. ${ }^{5}$ Although the exact mechanisms of systemic toxicity have still not been completely elucidated, previous studies suggest that jellyfish venoms contain neurotoxic, cardiotoxic, and hemolytic components that may lead to death from respiratory failure, cardiac failure, or anaphylactic shock. ${ }^{2,5,6}$ The severity of a jellyfish sting varies according to the species of jellyfish, degree of envenomation, and age and baseline health of the victim.

Various recommendations exist in the medical and lay literature regarding the most effective treatments for people who have experienced jellyfish stings. Some treatments seek to prevent the firing of undischarged nematocysts; others attempt to inactivate the injected toxin or decrease inflammation. Anecdotal recommendations and prior studies have referenced a wide variety of topical remedies, including such substances as human urine, vinegar, isopropyl alcohol, distilled spirits, ammonia, bleach, acetone, sodium bicarbonate, lidocaine, meat tenderizer, Coca-Cola, old wine, salt water, cold packs, hot packs, hot water, and commercial products such as Stingose or Stingaid..$^{2-5,7-12}$

Many sources recommend a single treatment method for all jellyfish stings, but it is likely that no single topical approach is effective for all known stinging species. For example, studies showing that acetic acid inhibits nematocyst discharge in multiple species of cubozoan jellyfish have cemented the role of acetic acid in the acute treatment of stings by the deadly Chironex fleckeri. ${ }^{6,8}$ However, studies using other jellyfish species have found that acetic acid causes nematocyst discharge $^{4,6-8}$ Conflicting data regarding nematocyst discharge have even been found for two species of Physalia. For instance, some studies utilizing the genus Physalia have observed nematocyst discharge after application of acetic acid, whereas others have found inhibition of discharge. ${ }^{7,9}$ Whether nematocyst discharge observed during in vitro experimentation actually translates to clinical outcomes is unknown.

In our study, we set out to create a human model of jellyfish sting treatment that would allow for testing of commonly proposed topical remedies in a controlled, prospective, randomized, and blinded manner. The human model was run in comparison with the in vitro model to assess for any correlation between clinical outcomes with nematocyst discharge triggered by topical treatments. This model would hopefully allow future studies to use the same or similar methodology.

The specific questions we sought to address were 1) which treatments increase or decrease the number of nematocysts discharged when viewed microscopically; 2) which treatments decrease pain in humans using a verbal pain scale; 3) which treatments decrease the amount of skin inflammation using an erythema index; and 4) whether there is a clinically relevant correlation between stimulated nematocyst discharge observed in vitro and the pain and erythema humans stung by a jellyfish experienced.

\section{Methods}

\section{JELLYFISH ACQUISITION}

We used Chrysaora chinensis (Figure 1), a tropical jellyfish species indigenous to coastal waters in the Andaman, Java, and South China Seas. This species of jellyfish was chosen for its ready availability and stinging potential. The jellyfish were supplied and maintained by the jellyfish husbandry team at the Monterey Bay Aquarium (MBA) in Monterey, California. $C$ chinensis jellyfish were shipped to the

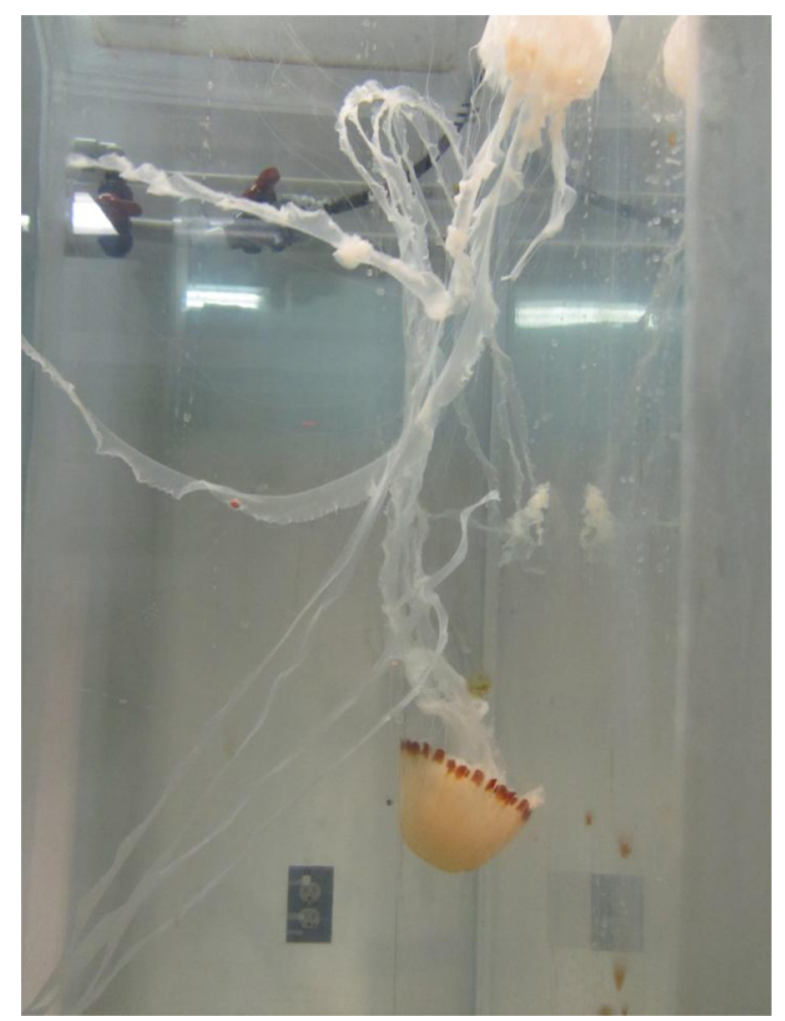

Figure 1. Live specimen of Chrysaora chinensis used for harvesting of tentacles for study. The jellyfish were housed in saltwater aquariums at the Monterey Bay Aquarium in California. 


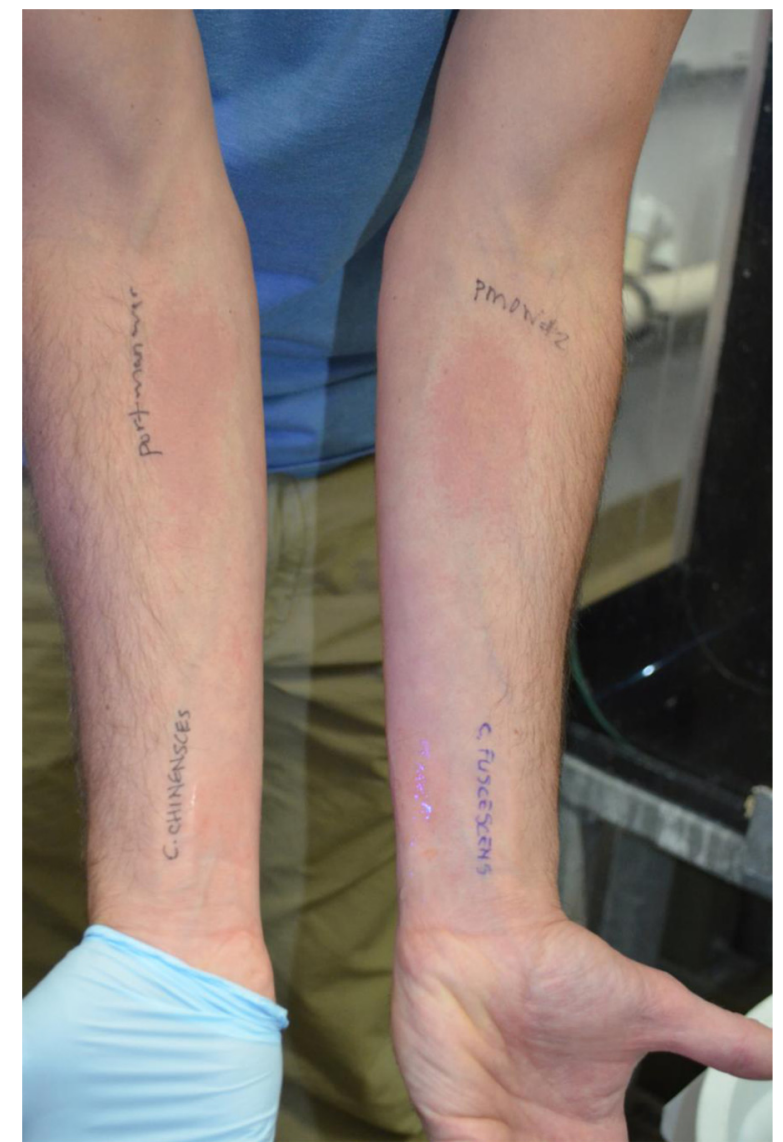

Figure 2. One of the author's forearms during testing phase of different species of jellyfish suitable to use for human study. Portuguese Man-of-War (Physalia physalis) had the most potent sting (top 2 stings) but was logistically difficult to obtain and maintain in captivity.

MBA from Sea Garden Aquarium in Penang, Malaysia. MBA provided the storage and maintenance of the jellyfish over the course of the month-long experiment free of charge. Wild-type, rather than cultured specimens, were used to ensure venom potency. The painful stinging potential of these $C$ chinensis was verified by 3 of the authors by placing a $2-\mathrm{cm}$ segment of oral arm on a bare forearm and mechanically exacerbating a sting by rubbing the tentacle. This preliminary testing was also performed with Physalia physalis, Chrysaora fuscescens, and Cassiopea xamachana (Figure 2). Although $P$ physalis tentacle showed the most potent sting, we were unable to ensure an adequate supply of jellyfish, which was compounded by the fragility of this species during shipment and captivity beyond 2 days. Neither $C$ fuscenscens nor $C$ xamanchana oral arm provided a sufficiently painful sting. $C$ chinensis oral arm was chosen because it provided a clinically significant sting and was reliably attainable, economically affordable, and relatively easy to maintain.
Jellyfish oral arm from $C$ chinensis were collected 2 to 3 times per week from the MBA by removing 20- to 30$\mathrm{cm}$ lengths of whole oral arm from live species. The detached segments were transported in glass containers filled with clean seawater from the MBA to the study site at Stanford University Hospital. The segments were stored at room temperature in glass containers and used for the study no later than 2 days postcollection. On each day of the study, 2-cm segments were cut and stored in a plastic 24-well plate (BD Falcon) containing seawater.

\section{HUMAN SUBJECTS}

The Stanford Institutional Review Board approved this study under the direction of Stanford University's Research Compliance Office. Healthy human subjects between the ages of 18 and 65 years were recruited by advertisement. We established an initial goal of enrolling approximately 15 subjects per treatment group (total 105) on the basis of financial and logistical considerations. Study participants were compensated for participation. Informed consent was obtained. Participation exclusion criteria were pregnancy, history of allergic reaction or anaphylaxis to prior Cnidaria or Hymenoptera sting, family history of anaphylaxis to Cnidaria or Hymenoptera sting, and history of cardiac or pulmonary disease.

\section{STING PHASE}

Each treatment episode was randomly assigned a singleletter identifier to blind both the researchers and subjects to therapies. No subjects were used for more than a single treatment exposure. Subjects randomly chose a treatment by picking an unmarked envelope and handing it to an investigator who was not actively applying treatment. The treatment letter and subject number were noted in paper records and marked on the subject's arm with a permanent marker (Figure 3). The subject was seated in a chair with his or her arms supported in a resting position. Both forearms were cleaned with a $70 \%$ isopropyl alcohol wipe (Covidien Webcol). Next, an $20.32 \mathrm{~cm} \times 15.24 \mathrm{~cm}$ Tegaderm (3M, St. Paul, MN) occlusive dressing with a $2.5-\mathrm{cm}$ opening cut out of the center was placed on the ventral surface of both forearms. This was performed to isolate the sting to a predetermined surface area of skin. A blindfold and nose clip were placed on the subject to ensure that no visual or olfactory stimuli cues from the treatment would bias the subject.

The opening in the occlusive dressing was outlined with a permanent marker for reference when recording erythema at 24-hour follow-up. Subjects were stung by placing a precut $2-\mathrm{cm}$ segment of oral jellyfish tentacle 


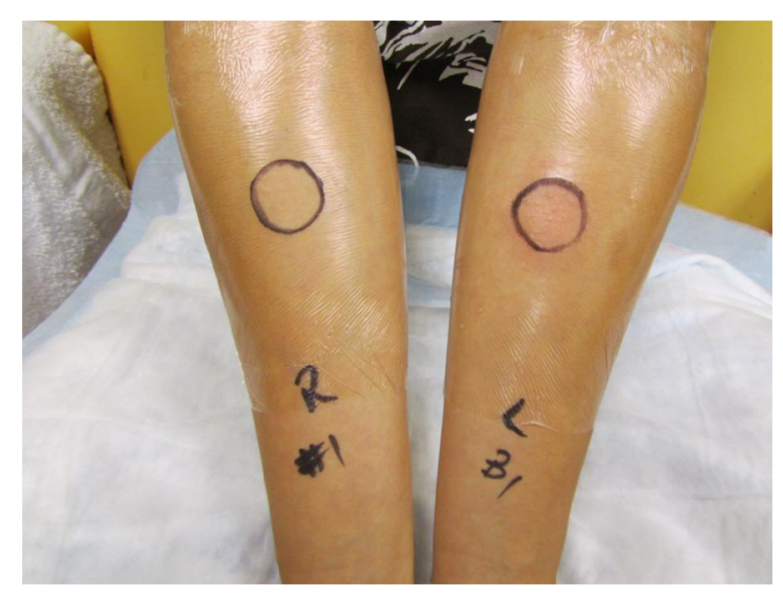

Figure 3. Participants' forearms, covered with occlusive dressing with $2.5-\mathrm{cm}$ hole cut out and marked. Forearms are labeled with participants number (1), treatment group (B1), and anatomical reference $(\mathrm{R}=$ right; $\mathrm{L}=$ left). The right forearm was the placebo (no treatment), extremity and the left forearm was the treatment extremity.

in the center of the marked circle on each arm. An investigator gently stimulated these segments by rubbing them in a circular fashion with gloved fingers for 2 minutes. After 2 minutes, the tentacle segments were removed from the subject's skin and discarded. The subjects were asked to numerically rate the most painful initial sting as a 5 and to rate subsequent pain measurements on the basis of that initial score. All subsequent pain measurements were then rated on a scale of 0 to 10 from the initial pain of 5 and noted as decreasing (less than 5) or increasing (greater than 5). The use of this unconventional pain score was adapted during the early phase of the experiment because some of the participants reported low initial pain scores. We initiated the anchoring of the initial pain score at a level of 5 to establish a measurable pain level to which subsequent pain levels could be compared.

\section{TREATMENT PHASE}

Each subject was told that different treatments were going to be applied to each arm with a specific script: "Some treatments might be wet, some might be pastelike, and some might be dry." They were neither informed of the specifics of the possible treatments nor that one arm would receive a control treatment. Subjects were randomly treated with either treatment A: isopropyl alcohol 70\% (Sigma-Aldrich); treatment B: ammonia $10 \%$ (Sigma-Aldrich); treatment $\mathrm{C}: 40^{\circ} \mathrm{C}$ tap water; treatment D: acetic acid 5\% (Sigma-Aldrich); treatment E: papain meat tenderizer 50\% slurry (Adolph's Meat Tenderizer); treatment F: liquid lidocaine 4\% (Morton Grove Pharmaceuticals); or treatment G: sodium

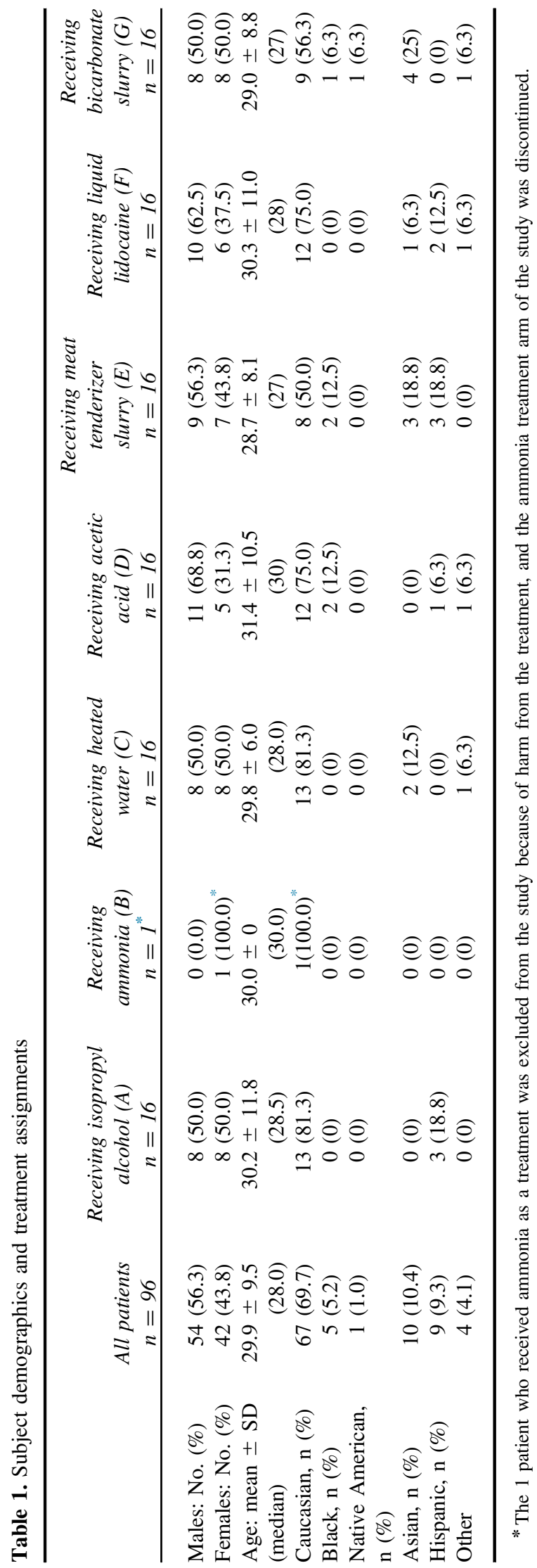


bicarbonate 50\% slurry (Sigma-Aldrich) (Table 1). All treatments were applied at room temperature except for the heated water, which was kept at a temperature of $40^{\circ} \mathrm{C}$ using a standard laboratory water bath (Thermo Scientific). The concentrations of treatments used were based either on concentrations used in previous studies or on the standard concentrations found in commercially available products.

In all subjects, the left forearm was the treatment arm and the right forearm was the control arm. A $5.08 \mathrm{~cm} \times$ $5.08 \mathrm{~cm}$ piece of gauze (Covidien Curity) was soaked in the predetermined treatment and placed on the subject's left forearm over the stung area, and a dry piece of gauze was placed over the stung area of the subject's right arm. Treatment was performed immediately after recording the subject's the initial pain score for the sting. The gauze was allowed to sit on the subject's arm for 2 minutes, at which point the subject's pain scale was again reported for each arm. At 2 minutes, for the treatment side, an immediate reapplication of $5 \mathrm{~mL}$ of treatment substance was made by lifting the gauze, rewetting it, and replacing the gauze on the left arm. At the same time, the control gauze on the right arm was also lifted and replaced. This 2-minute cycle of evaluating a subject's pain score and reapplying treatment was repeated 15 times for a total of 30 minutes. Treatment B (ammonia 10\%) was removed from the study because of an adverse reaction described further in the Results section.

After 30 minutes of treatment, the gauze, blindfold, and nose clamp were removed, and a picture was taken of each subject's forearms side by side (Canon SX210 IS, Tokyo, Japan) (Figure 3). The occlusive dressings remained on each subject's arms, and they were instructed to wait another 30 minutes. At 60 minutes poststing, subjects' pain scores for each arm and a picture of their forearms were again recorded. At this point, they were instructed to remove the occlusive dressing and to wash their forearms with warm, soapy water. Telephone follow-up was done the next day (approximately 24 hours poststing), during which the subject's pain score for each arm was recorded a final time. Subjects reported on the lingering erythema by noting whether "the area of redness on each arm is smaller, larger, or the same size compared to the permanent marker circle placed the previous day." All data were recorded on a master collection sheet.

Pain was rated on the previously described verbal scale for each arm every 2 minutes at times $(\mathrm{t})=2,4,6$, $8,10,12,14,16,18,20,22,24,26,28$, and 30 minutes. Pain was again rated on the same scale at $\mathrm{t}=60$ minutes and $t=24$ hours. Multiple measurements were recorded across time to capture any trends in response to the topical treatments.

\section{ERYTHEMA INDEX}

We followed the methods delineated by Yamamoto et al using a computer model to establish an erythema index (EI) that quantifies the amount of erythema on human skin. ${ }^{13}$ EI is a method of measuring an increase in cutaneous vasodilation independent of the melanin content of the epidermis; its scale ranges between negative and positive infinity, with 0 indicating equal red light reflectance and green light reflectance by the skin, positive values indicating more red light reflectance, and negative values indicating more green reflectance. ${ }^{14} \mathrm{~A}$ computer was used to measure EI to remove any subjectivity that might occur from measurements using the human eye. Image-J software version 1.47 was used to isolate the color red and quantify the index from images taken of the subjects' forearms poststing. ${ }^{15}$ The stung area was traced, selected, and analyzed. The region of interest was analyzed 3 separate times, and the mean EI was recorded. This analysis was performed in full for all available photographs at 30 minutes and 60 minutes poststing.

\section{NEMATOCYST DISCHARGE}

Oral arms from the jellyfish $C$ chinensis were collected from their housing unit at the MBA. They were transported in glass containers containing room temperature seawater to a laboratory at Stanford University Medical Center for immediate use. The tentacles were sectioned into 0.25 to $0.5 \mathrm{~cm}$ segments and placed into plastic 12 well plates (BD Falcon) accompanied by $0.5 \mathrm{~mL}$ of seawater per well. The plates were placed on an optical light microscope (Leica DMIL) for visualization. The seawater was aspirated from each well before visualization, and the tentacles were gently arranged in a flat configuration with forceps.

Using 1000-mL micropipettes (Gilson), $1 \mathrm{~mL}$ of each of the same treatment reagents used to treat the human subjects (with treatment $\mathrm{B}$ omitted) was added to separate wells containing jellyfish sections. We observed immediate nematocyst discharge upon addition of acetic acid, whereas discharge with isopropyl alcohol progressed at a much slower rate. Isopropyl alcohol induced active nematocyst discharge up to 35 minutes after addition. The other reagents induced no significant discharge. Based on this observation, each reagent was allowed to interact with the jellyfish sections for 45 minutes before the number of discharged versus undischarged nematocysts were counted.

After 45 minutes elapsed, the jellyfish tissue was examined at $\times 40$ magnification to identify clusters of countable nematocysts. Clusters were defined as groups 


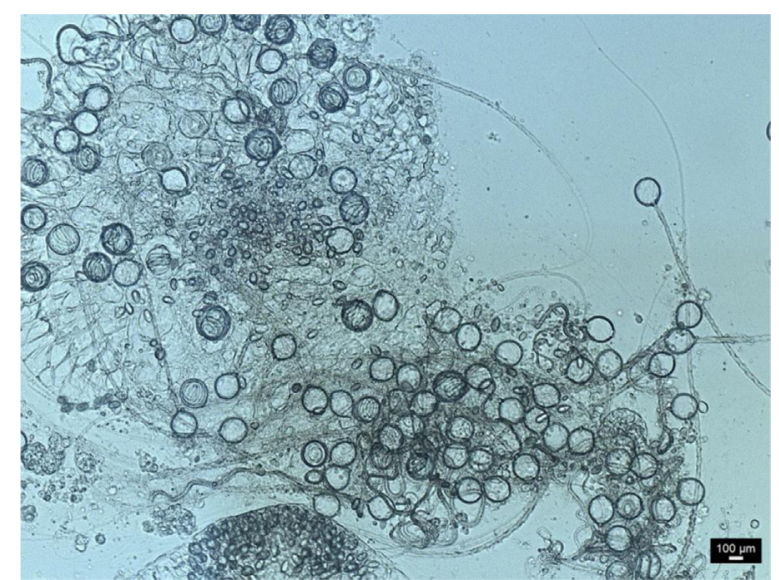

Figure 4. Fired and unfired nematocysts seen at $\times 200$ magnification. This grouping of nematocyst was defined as a "cluster" for the in vitro analysis.

of multiple, large, round nematocysts found in close spatial proximity to each other within the field of vision (Figure 4). The nematocysts within these clusters were evaluated individually at $\times 200$ or $\times 400$ magnification. In each cluster, the numbers of discharged and undischarged nematocysts were recorded before and after the addition of each reagent (Figure 5). Because some clusters had a greater number of nematocysts than others, 10 to 12 clusters were evaluated for each reagent to obtain the averages. This experiment was repeated in its entirety again on a second day. Jellyfish oral arm tissue left in plain seawater for the entire 8-hour duration of the experiment served as a negative control and showed no nematocyst discharge.

\section{STATISTICAL ANALYSIS}

For the human subjects, the following outcomes were recorded by subject and forearm: mean pain score reported over the first 30 minutes of treatment, maximum pain score reported over the first 30 minutes of treatment, and erythema index at 30 and at 60 minutes after start of treatment. No pre-experimental power analysis was performed.

Linear mixed effects models were estimated for mean pain and maximum pain scores, with fixed effects for treatment and random effects by subject; the random effects account for correlations between data from the right and left forearms of the same subjects. The 'nlme' (Linear and Nonlinear Mixed Effects Models) package for $\mathrm{R}$ was used to estimate these models, using the restricted maximum likelihood method. Wald tests and confidence intervals were used to compare each of the active treatments with placebo. Because both positive and negative effects were of interest, 2-sided tests were used.

Linear mixed effects models were used to model EI at 30 minutes and at 60 minutes, with fixed effects for treatment and random intercepts by arm-nested-in-subject, to account for the 3 repetitions of region of interest selection and the pairing of placebo and treatment data for each subject. Similar to the analysis of pain, restricted maximum likelihood estimation was used and compared each active treatment with placebo, again using 2-sided Wald tests and confidence intervals. This analysis provides estimates of the difference in mean EI between each treatment and placebo; positive values of these estimates indicate that a treatment increases EI relative to placebo, and negative values indicate that a treatment decreases EI relative to placebo.

For the in vitro nematocyst discharge data, Fisher exact test was used to compare each reagent with the

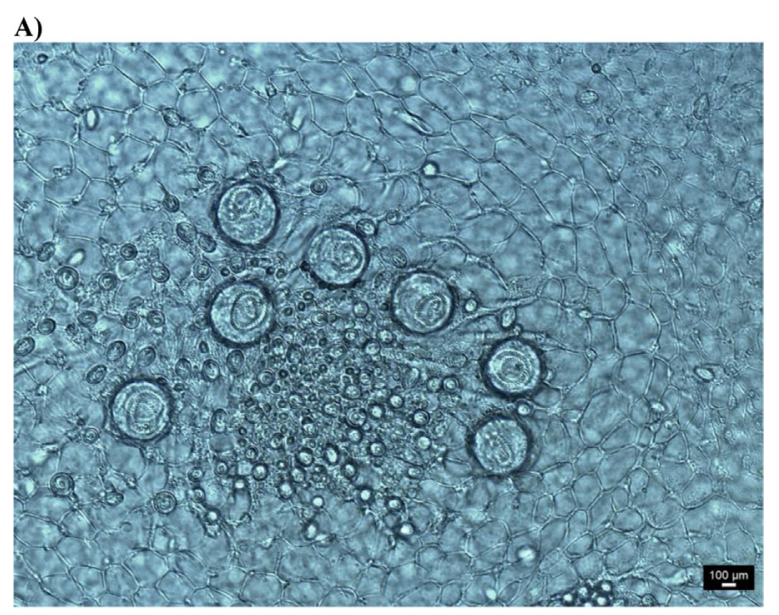

B)

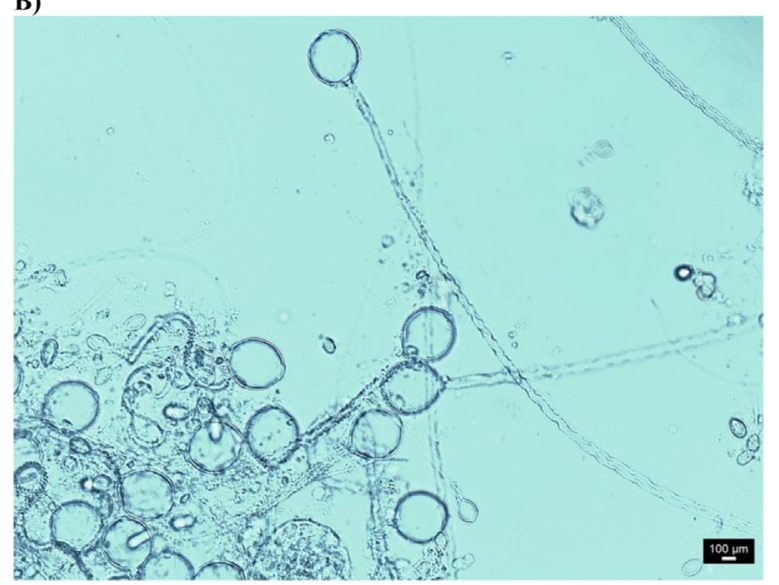

Figure 5. Nematocyst light microscopy: (A) Unfired nematocysts (visible as circles) at $\times 400$ magnification. (B) Fired nematocysts (visible as circles with protruding linear appendages) at $\times 400$ magnification. 
control (seawater) and the other reagents. The variance in discharge rate between reagents was much larger than the variance between clusters of nematocysts exposed to the same reagents. Therefore, to simplify the analyses, the tests were not adjusted for the possibility of correlations between nematocysts from the same cluster.

The data were analyzed using $\mathrm{R}$ version 3.0.2. Graphics were produced using the lattice package for R. ${ }^{16}$

\section{Results}

Ninety-seven subjects were stung in this study. One subject was excluded from the study because she experienced a first-degree chemical burn attributed to treatment with ammonia 10\% (Treatment B). Ammonia was therefore discontinued as an experimental treatment, and data collected from this subject were not included in the final analysis of data and results. The subjects slated for the ammonia treatment group were rerandomized to the other treatment groups, resulting in 6 treatment groups with 16 subjects each. Data from the remaining 96 subjects were analyzed. Of these, 16 were treated with isopropyl alcohol, 16 with heated water, 16 with acetic acid, 16 with papain meat tenderizer, 16 with lidocaine, and 16 with sodium bicarbonate. The subjects' demographic information is provided in Table 1.

\section{REPORTED PAIN}

Reported pain was highly variable between subjects and somewhat variable between treatments (Figure 6). Variance in mean pain between subjects was 1.16 , and variance between treatments was 0.12. Overall, time elapsed from start of treatment was a major factor in reported pain scores; average pain score fell substantially for all treatments as the time after the initial exposure increased. Pain scores recorded at 60 minutes poststing among the majority of subjects were negligible and thus not included in the final analysis.

\section{PAIN BY TREATMENT}

Assuming a linear mixed effects model for mean reported pain over the first 30 minutes after jellyfish sting, there is statistically significant $(P=.0424)$ evidence that forearms treated with papain had less pain on average than forearms treated with placebo by an estimated 0.46 on the pain scale reported over 30 minutes (Table 2). Although the data showed trends of increased mean pain in stings treated with heated water (by 0.43 points; $P=.059$ ) and acetic acid (by 0.13 points; $P=.554$ ), these findings were not statistically significant. Similarly, although the data showed trends of decreased mean pain in stings treated with isopropyl alcohol (by 0.27 points; $P=.237$ ), lidocaine (by 0.32 points; $P=.159$ ), and sodium bicarbonate (by 0.19 points; $P=.398$ ), these findings were not statistically significant.

Assuming a linear mixed effects model for maximum reported pain, none of the treatments was observed to be significantly different from placebo with regard to maximum pain reported over 30 minutes (Table 3). Although the data showed trends of increased maximum pain in stings treated with heated water (by 0.17 points; $P=.597$ ) and acetic acid (by 0.43 points; $P=.169)$ compared with stings treated with placebo, these findings were not statistically significant. Similarly, although the data showed trends of decreased maximum pain in stings treated with isopropyl alcohol (by 0.16 points; $P=.603$ ), papain (by 0.51 points; $P=.108$ ), lidocaine (by 0.33 points; $P=.290$ ), and sodium bicarbonate (by 0.25 point; $P=.423$ ) compared with stings treated with placebo, these findings were not statistically significant.

\section{ERYTHEMA INDEX}

We found statistically significant evidence that sodium bicarbonate reduces EI after 30 minutes of treatment by an estimated $12 \mathrm{EI}$ units $(P=.0013)$ (Table 4$)$. There was also statistically significant evidence that both sodium bicarbonate and papain reduced EI at 60 minutes, the former by 16.5 units $(P<.0001)$ and the latter by 8.5 units $(P=.0125)$ (Table 5$)$.

Measured at 30 minutes, EI showed trends of increased erythema in skin treated with isopropyl alcohol (by 2.11 units; $P=.599$ ), acetic acid (by 4.43 units; $P=.271$ ), and papain (by 0.59 units; $P=.884$ ) compared with skin treated with placebo (Table 4). Similarly, there were trends of reduced erythema at 30 minutes in skin treated with heated water (by 4.11 units; $P=.289$ ) and lidocaine (by 4.15 units; $P=.269$ ). None of these trends was statistically significant.

Measured at 60 minutes, EI showed trends of increased erythema in skin treated with heated water (by 0.77 units; $P=.812$ ) (Table 5). Similarly, there were trends of decreased erythema in skin treated with isopropyl alcohol (by 4.34 units; $P=.165$ ), acetic acid (by 0.72 units; $P=.817$ ), and lidocaine (by 0.99 units; $P=.741)$. None of these trends was statistically significant.

\section{4-HOUR FOLLOW-UP}

At the 24-hour follow-up call, all subjects reported a substantial decrease in or complete resolution of pain and erythema at the sites of both stings. There was no 


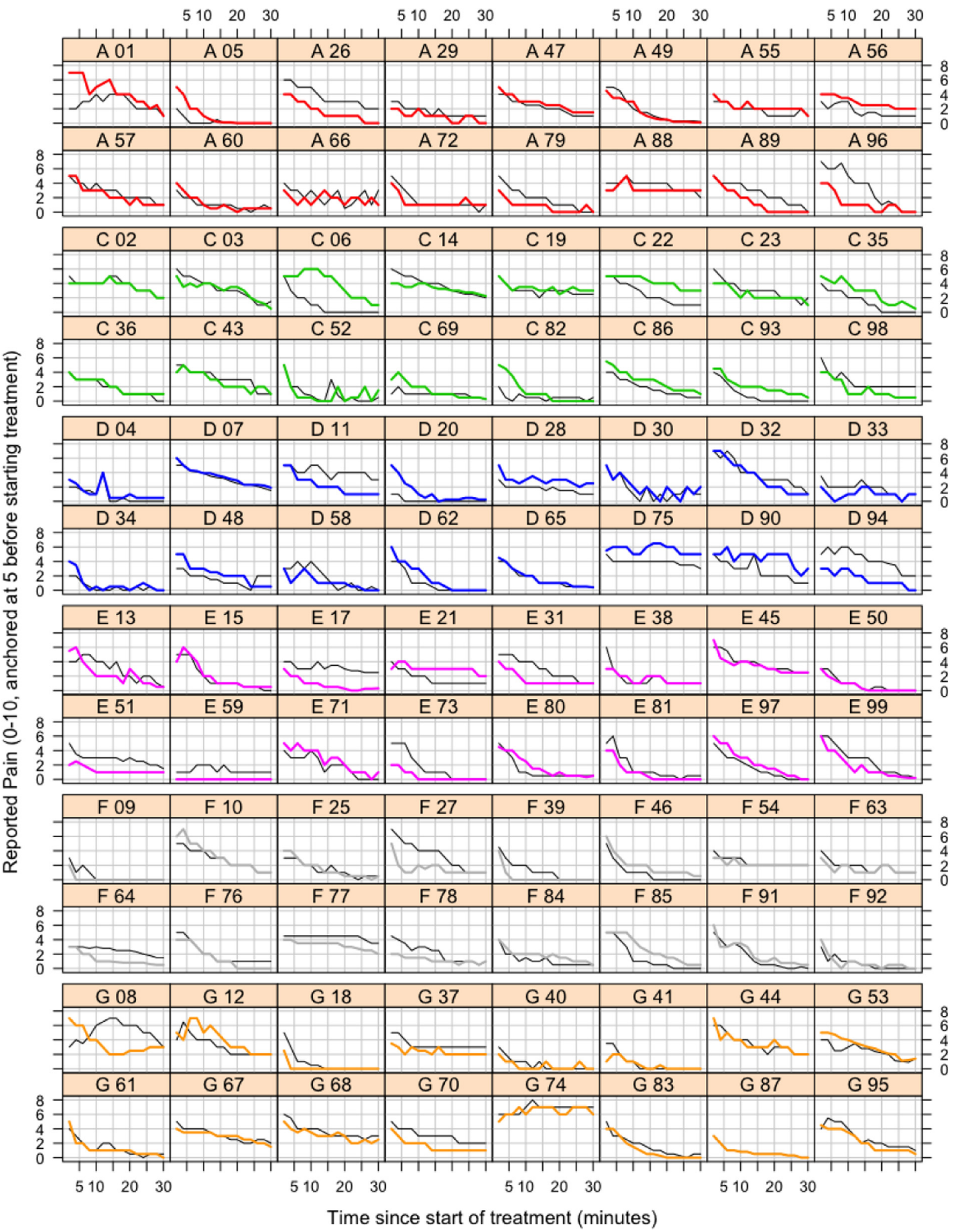

Figure 6. Pain of both arms vs time, grouped by subject. Pain over time, grouped by subject. The thick black lines show active treatments; the thin gray lines show placebo treatments. 
Table 2. Treatment effects on mean pain

\begin{tabular}{|c|c|c|c|c|}
\hline & Estimated effect & $95 \%$ CI low & $95 \%$ CI high & $P$ value \\
\hline (Intercept) & 2.1790 & 1.9413 & 2.4167 & $<.0001$ \\
\hline Treatment A: isopropyl alcohol & -0.2650 & -0.7071 & 0.1772 & .2370 \\
\hline Treatment C: heated water & 0.4246 & -0.0176 & 0.8667 & .0597 \\
\hline Treatment D: acetic acid & 0.1322 & -0.3100 & 0.5744 & .5541 \\
\hline Treatment E: papain & -0.4583 & -0.9005 & -0.0161 & .0424 \\
\hline Treatment F: lidocaine & -0.3161 & -0.7583 & 0.1260 & .1590 \\
\hline Treatment G: baking soda & -0.1892 & -0.6314 & 0.2530 & .3976 \\
\hline
\end{tabular}

CI, confidence interval.

difference in pain or erythema between the treatment groups at 24 hours.

\section{NEMATOCYST DISCHARGE IN VITRO}

Substantial in vitro nematocyst discharge can be reliably elicited when tentacles of $C$ chinensis are exposed to acetic acid (89\% discharge; $P<.001$ compared with seawater, which showed $0 \%$ discharge) or isopropyl alcohol (94\% discharge; $P<.001$ compared with seawater; not statistically different from acetic acid discharge, $P>.05)$. Sodium bicarbonate (2\% discharge), papain ( $2 \%$ discharge), and lidocaine (1\% discharge) did not induce substantial nematocyst discharge and were not statistically distinguishable from seawater $(P>.05)$. Heated water (4\% discharge) did not induce substantial discharge but was statistically different from seawater $(P=.02)$ (Table 6 and Figure 7$)$.

\section{Limitations}

The greatest limitation to our study was that a larger number of subjects than we enrolled, a larger treatment effect, and/or a treatment effect that varied less among subjects was needed to reliably attain statistical significance (see Recommendations). We encountered logistical limitations in enrolling a number of subjects large enough to investigate 6 treatments to the extent that we could draw statistically significant conclusions for each treatment. Looking at a single treatment rather than 6 different treatments for the number of subjects enrolled would have substantially improved the power of our data (see Recommendations). No pre-experimental power analysis was performed. This would have been beneficial in determining how many treatments could be reasonably evaluated with the number of subjects recruited.

We attempted to create a built-in control by using each subject's right forearm as the untreated control to which they could compare the treated left forearm. It is possible that being stung or treated with a painful topical treatment on one forearm could influence the pain felt on the contralateral arm, an effect referred to as diffuse noxious inhibitory control. ${ }^{17}$ The potential for the diffuse noxious inhibitory control effect might have been avoided by a crossover study design in which the control forearm was stung at a different time than the treatment forearm.

This study looked at a single species of jellyfish $(C$ chinensis). There are many morphological types of nematocysts and a wide variation of clinical manifestations between different species of jellyfish. Although this experimental model can be reproduced using different species of jellyfish, our findings can only be correlated to stings caused by $C$ chinensis.

Table 3. Treatment effects on maximum pain

\begin{tabular}{|c|c|c|c|c|}
\hline & Estimated effect & $95 \%$ CI low & $95 \%$ CI high & $P$ value \\
\hline (Intercept) & 4.5469 & 4.2735 & 4.8203 & $<.0001$ \\
\hline Treatment A: isopropyl alcohol & -0.1640 & -0.7875 & 0.4594 & .6025 \\
\hline Treatment $\mathrm{C}$ : heated water & 0.1693 & -0.4542 & 0.7927 & .5910 \\
\hline Treatment D: acetic acid & 0.4349 & -0.1885 & 1.0584 & .1692 \\
\hline Treatment E: papain & -0.5099 & -1.1334 & 0.1136 & .1077 \\
\hline Treatment F: lidocaine & -0.3338 & -0.9573 & 0.2896 & .2903 \\
\hline Treatment G: baking soda & -0.2527 & -0.8761 & 0.3708 & .4229 \\
\hline
\end{tabular}

Estimated effects on maximum pain by candidate treatments compared with placebo and adjusted for random effects by subject. Negative effect estimates indicate reduced pain.

CI, confidence interval. 
Table 4. Treatment effects on erythema index at 30 minutes

\begin{tabular}{|c|c|c|c|c|}
\hline & Estimated effect & $95 \%$ CI low & $95 \%$ CI high & $P$ value \\
\hline Treatment A: isopropyl alcohol & 2.1128 & -5.8483 & 10.0739 & .5988 \\
\hline Treatment $\mathrm{C}:$ heated water & -4.1128 & -11.7888 & 3.5631 & .2894 \\
\hline Treatment D: acetic acid & 4.4342 & -3.5269 & 12.3952 & .2709 \\
\hline Treatment E: papain & 0.5867 & -7.3744 & 8.5477 & .8837 \\
\hline Treatment F: lidocaine & -4.1487 & -11.5687 & 3.2713 & .2691 \\
\hline Treatment G: baking soda & -12.0738 & -19.2623 & -4.8853 & .0013 \\
\hline
\end{tabular}

Estimated effects on erythema index at 30 minutes by candidate treatment compared with placebo and adjusted for random effects by treatment nested in subject. Negative effect estimates indicate reduced erythema index.

CI, confidence interval.

Our study was hampered by the low degree of pain experienced by the human subjects. Participants sometimes reported that the sting from $C$ chinensis did not induce significant pain, and it was sometimes difficult to differentiate between pain felt on the respective forearms. Similarly, the stings only induced moderate erythema. In summary, the relative weakness of the sting caused by $C$ chinensis made it difficult to assess for differences in pain felt by subjects, both between the forearms of individuals and between treatment groups. Future studies may be able to avoid this phenomenon by using other species of jellyfish and methods of preservation and promptly applying harvested nematocysts to ensure stings that generate more intense pain and erythema.

We used segments of oral arm from $C$ chinesis rather than the tentacle because oral arm was larger and easier to handle than the rather fine tentacle of this species. Oral arm nematocysts differ morphologically from tentacle nematocysts, are fewer in number, and may be less potent. Stings in humans typically result from tentacles rather than oral arms, and this may also have led to a weaker sting than expected. Future studies would benefit from using tentacle segments alone rather than oral arm segments.

Jellyfish oral arms were used up to 2 days after the time of collection, which may have altered the potency and consistency of the stings because of degradation. Fresher specimens are easier to handle because they better retain structural integrity during manipulation. Using specimens immediately after harvesting may also enhance the intensity and uniformity of the skin reactions.

Anchoring the pain scale at 5 may have artificially amplified low levels of pain and undercut higher levels of pain, creating a nonstandard pain scale, which was in reality a 5-unit rather than a 10-unit scale. Amplifying lower levels of pain to a set point of 5 may have inflated noise and created unreliable data. Future studies may use standardized pain scales that have been validated in prior trials, such as a visual analog scale or other visual pain scale. Using a species of jellyfish that is more potent may also obviate the need to use a manipulated pain scale.

Subjects were blinded to the treatments. Although the researchers were theoretically blinded to the treatments, they likely could distinguish which treatment was being used on the basis of the mode of delivery and by the unique physical qualities of the treatments. Using dry gauze as the control treatment may have caused unblinding of the subjects. Use of seawater-soaked gauze as a control treatment could resolve this limitation.

For 1 category of treatment, we applied a small volume of heated water every 2 minutes. This likely allowed the applied heated water to cool to ineffective temperature between applications. In the future, it may

Table 5. Treatment effects on erythema index at 60 minutes

\begin{tabular}{|c|c|c|c|c|}
\hline & Estimated effect & $95 \%$ CI low & $95 \%$ CI high & $P$ value \\
\hline Treatment A: isopropyl alcohol & -4.3392 & -10.4987 & 1.8203 & .1647 \\
\hline Treatment C: heated water & 0.7673 & -5.6214 & 7.1561 & .8116 \\
\hline Treatment D: acetic acid & -0.7178 & -6.8773 & 5.4417 & .8171 \\
\hline Treatment E: papain & -8.5356 & -15.1818 & -1.8894 & .0125 \\
\hline Treatment F: lidocaine & -0.9902 & -6.9439 & 4.9635 & .7414 \\
\hline Treatment G: baking soda & -16.5158 & -22.6754 & -10.3563 & $<.0001$ \\
\hline
\end{tabular}

Estimated effects on erythema index at 60 minutes by candidate treatment compared with placebo and adjusted for random effects by treatment nested in subject. Negative effect estimates indicate reduced erythema index.

CI, confidence interval. 
Table 6. Stimulation of nematocyst discharge

\begin{tabular}{|c|c|c|c|c|c|c|}
\hline \multirow[b]{2}{*}{ Reagent } & \multirow[b]{2}{*}{ Number discharged } & \multirow[b]{2}{*}{ Number not discharged } & \multirow[b]{2}{*}{ Number total } & \multirow[b]{2}{*}{ Percent discharged } & \multicolumn{2}{|c|}{$95 \% C I$} \\
\hline & & & & & Low & High \\
\hline Acetic acid & 247 & 31 & 278 & $88.80 \%$ & $85 \%$ & $92 \%$ \\
\hline Bicarbonate & 5 & 237 & 242 & $2.10 \%$ & $1 \%$ & $5 \%$ \\
\hline Hot water & 13 & 305 & 318 & $4.10 \%$ & $2 \%$ & $7 \%$ \\
\hline Isopropyl alcohol & 234 & 16 & 250 & $93.60 \%$ & $90 \%$ & $96 \%$ \\
\hline Lidocaine & 3 & 291 & 294 & $1.00 \%$ & $0 \%$ & $3 \%$ \\
\hline None (dry) & 2 & 115 & 117 & $1.70 \%$ & $0 \%$ & $6 \%$ \\
\hline Papain & 7 & 326 & 333 & $2.10 \%$ & $1 \%$ & $4 \%$ \\
\hline Seawater & 0 & 123 & 123 & $0.00 \%$ & $0 \%$ & $3 \%$ \\
\hline
\end{tabular}

Nematocysts were exposed in vitro to a variety of treatments, and nematocyst discharge data were collected by light microscopy. Our results indicate that acetic acid and isopropyl alcohol induce significant nematocyst discharge, whereas sodium bicarbonate, papain, lidocaine, and heated water do not. Jellyfish oral arm tissue left in plain seawater for the entire 8-hour duration of the experiment served as a negative control and showed no significant amount of nematocyst discharge.

CI, confidence interval.

be more effective to provide a steady source of heated water to maintain the treatment temperature. The application of hot water as a treatment likely causes local vasodilation in the skin and could potentially cause higher determinations of immediate EI measurements. This effect should be considered in the time of EI measurements for future studies but was likely dissipated by 30 and 60 minutes when measurements were taken in this study.

Because of the lack of a white standard when measuring EI, we were unable to white balance our images as described in Yamamoto et al. ${ }^{13}$ Hence, the derived erythema indices are not optimally standardized. Variations in ambient lighting, photographic technique, and image quality may have affected standardization of the EI. Despite wiping, the bicarbonate and papain slurries may have left some white residue on the skin surface. This residue might have occluded erythema captured on the photograph, resulting in falsely low determination of EI. For future studies, the observed skin should be gently rinsed before photographs are taken. This was not performed in this study because of concern

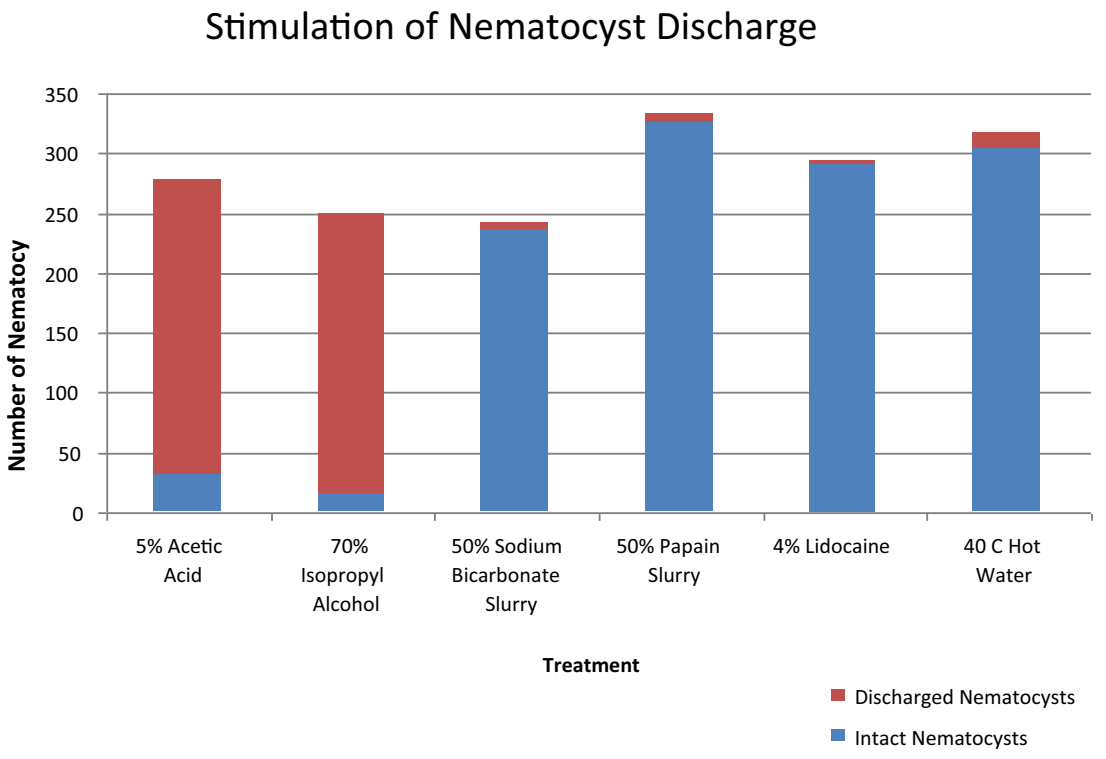

Figure 7. Stimulation of nematocyst discharge. Nematocysts were exposed in vitro to a variety of treatments, and nematocyst discharge data were collected by light microscopy. Our results indicate that acetic acid and isopropyl alcohol induce significant nematocyst discharge, whereas sodium bicarbonate, papain, lidocaine, and heated water do not. Jellyfish oral arm tissue left in plain seawater for the entire 8-hour duration of the experiment served as a negative control and showed no significant amount of nematocyst discharge. 
that fully rinsing off the treatment at time 30 may alter the pain score at 30 and 60 minutes by removing the therapeutic effect of the slurries.

\section{Discussion}

This study was conceived in response to the concern embodied in M. Little's request that "national and international recommendations for first aid treatment of jellyfish stings be supported by evidence." ${ }^{9}$ We recognized that this might be difficult in light of inherent variations in the biological activity of individual jellyfishes and responsiveness of human subjects.

Our data show statistically significant evidence that topical application of papain-containing meat tenderizer in the form of a slurry to the sting of a $C$ chinensis jellyfish decreased mean pain over the first 30 minutes after being stung, relative to placebo, although only by a small amount. We want to caution the reader that this experimental measurement may not be significant enough to translate into clinical relevance. Furthermore, pain scores recorded at 60 minutes poststing were negligible in the majority of subjects for both arms. This leads to an important observation for this species of jellyfish: The pain felt from an initial sting is short lived and likely resolves spontaneously regardless of whether it is treated in a "real world" clinical setting.

Our data show that topical application of sodium bicarbonate to the sting of $C$ chinensis may reduce erythema on human skin measured at 30 minutes and 60 minutes poststing and that papain-containing meat tenderizer in the form of a slurry may reduce erythema on human skin observed at 60 minutes poststing. However, both papain and sodium bicarbonate were delivered in an opaque solution that left a whitish residue, which diminishes the reliability of this observation.

The generally accepted treatment approach for jellyfish envenomation seeks both to treat the effects of any venom already delivered in the victim and to inhibit any further envenomation. ${ }^{8}$ With regard to the latter goal, it is logical that removal of cnidarian tissue and prevention of nematocysts activation ("firing" or "discharge") would be expected to reduce venom delivery. In light of past studies showing in vitro nematocyst discharge with certain topical treatments, we wondered whether topical treatments that chemically trigger nematocyst discharge might make the sting worse by inducing further envenomation.

To help understand the relationship of nematocyst activity in vitro to clinical treatment in vivo, we performed an in vitro nematocyst discharge experiment to complement our human study (Table 6 and Figure 7). Our goal was to see whether a correlation exists between nematocyst discharge and pain and erythema experienced by humans when using topical treatments that either chemically induce or inhibit nematocyst discharge. Our methodology mirrored that of Birsa et al., who studied similar topical reagents for the treatment of Chrysaora and Physalia species. ${ }^{4}$ In agreement with that investigation, we found statistically significant evidence that acetic acid and alcohol both caused nematocyst discharge, although we note that the prior study used ethanol whereas we used isopropyl alcohol. However, we did not observe meat tenderizer to elicit nematocyst discharge, whereas the previous authors did. They used bromain, and we used papain. One could argue that there may be a correlation between the decreased pain observed in our human subjects treated with papain and the observation that papain did not trigger nematocyst discharge in vitro. The inhibition of further nematocyst discharge that would otherwise occur with continued exposure and manipulation of jellyfish tentacles on human skin might result in decreased pain and erythema scores. A true correlation of such relationship was not determined by our study because of the lack of statistical significance reached in the human study. However, this remains a theoretical question in the treatment of jellyfish stings.

In conclusion, although there is published experimental support for the concept that in vitro nematocyst discharge correlates with in vivo human pain perception, no definitive randomized controlled trial, including ours, has yet provided incontrovertible evidence of this assertion. ${ }^{4,18}$ Not all commenters accept that in vitro nematocyst activation necessarily equates to in vivo envenomation. For instance, it is possible that in vitro nematocyst activation caused by application of a decontaminant (eg, acetic acid or isopropyl alcohol) represents a terminal event for the nematocyst, which is thereafter incapable of functioning to envenom a human subject. Until it is determined precisely what occurs when a nematocyst changes its configuration by being exposed to a "treatment," it will be difficult to equate microscopically observed configuration changes with the effects upon clinical envenomation in humans. Until further notice, this lessens our confidence about in vitro observations and increases the importance of properly designed and valid in vivo studies.

\section{Recommendations}

Although we did not accomplish a definitive study for this jellyfish species and the treatments studied, we learned a sufficient amount to make recommendations that will hopefully guide future studies to success. Although our conclusions are limited, we suggest several possible factors important for a successful model to undertake a randomized, prospective, blinded, and 
controlled method for evaluation of pain and erythema in human subjects who have sustained a jellyfish sting. We recommend the following:

1. Future studies should improve statistical power by appropriately maximizing the number of subjects in each treatment arm. For example, to compare 1 experimental treatment with placebo, the mixed effects model for mean pain would be equivalent to a paired $t$-test. Assuming that the estimate of the difference between treatment and control for papain $(-0.458)$ is the true difference and that the standard deviation of that difference is 0.985 (estimated from our current data), one would need data from 39 subjects to statistically detect the observed difference at the 0.05 significance level with $80 \%$ power. To achieve $99 \%$ power at the .01 significance level, one would need data from 115 subjects. A model that looked at a single treatment compared with either placebo or a single other treatment would allow for the minimum number of enrollees to achieve reliable data that could be indicative of statistical significance.

2. Future studies should use a clinically relevant jellyfish species that delivers a sufficiently potent sting such that it can generate sufficient pain and erythema (or other skin reaction) to detect differences caused by treatment. Fresh, wild tentacle that can be applied immediately after harvest will ensure maximum sting potential for this experimental model.

3. Future studies should use a previously validated pain scale (score).

4. Future studies should use a method to measure erythema that is controlled for lighting, photographic method, and white balancing. Skin should be properly prepared to avoid confounding coloration.

5. Future studies should determine the biological significance of in vitro nematocyst discharge to determine whether it represents a single or multiple physiological activities. For instance, it needs to be known whether the act of deformation equates with venom injection.

6. Future studies may use this initial model as an aid in improving and ultimately developing a viable human model to shed light on what topical treatments truly provide clinically significant, evidence-based results for the treatment of jellyfish stings.

\section{Acknowledgments}

The authors thank Geoffrey Gurtner, MD for use of his laboratory, Sue Lisin of the Monterey Bay Aquarium, and Justine Samonte and Leslie Lew from the Division of Emergency Medicine at Stanford.

Statistical analysis was facilitated by Spectrum Services at the Stanford University School of Medicine. Spectrum is funded, in part, by the NIH Clinical and Translational Science Award (Grant UL1 TR000093).

This study was supported by a grant provided by the Diver's Alert Network (DAN) given to Paul S. Auerbach, MD, MS (senior author of this manuscript), from the Division of Emergency Medicine, Department of Surgery, at Stanford University School of Medicine. There were no stipulations placed on the use of the grant money, and DAN had no influence or involvement in the planning, execution, or publication of the study.

\section{References}

1. Dickerson, Nathan. Death by jellyfish sting. Jellyfish Facts. Available at: http://www.jellyfishfacts.net/death-byjellyfish-sting.html. Accessed September 1, 2014.

2. Auerbach PS. Envenomations by aquatic invertebrates. In: Auerbach PS, ed. Wilderness Medicine. 6th ed. Philadelphia, PA: Elsevier-Mosby; 2012:1596-1628.

3. Auerbach PS. Envenomations from jellyfish and related species. J Emerg Nurs. 1997;23:555-565. quiz 566-567.

4. Birsa LM, Verity PG, Lee RF. Evaluation of the effects of various chemicals on discharge of and pain caused by jellyfish nematocysts. Comp Biochem Physiol Toxicol Pharmacol. 2010;151:426-430.

5. Nimorakiotakis B, Winkel KD. Marine envenomations. Part 1-Jellyfish. Aust Fam Physician. 2003;32:969-974.

6. Bailey PM, Little M, Jelinek GA, Wilce JA. Jellyfish envenoming syndromes: unknown toxic mechanisms and unproven therapies. Med J Aust. 2003;178:34-37.

7. Tibballs J. Australian venomous jellyfish, envenomation syndromes, toxins and therapy. Toxicon. 2006;48:830-859.

8. Ward NT, Darracq MA, Tomaszewski C, Clark RF. Evidence-based treatment of jellyfish stings in North America and Hawaii. Ann Emerg Med. 2012;60: 399-414.

9. Little M. First aid for jellyfish stings: do we really know what we are doing? Emerg Med Australas. 2008;20: 78-80.

10. Burnett JW, Rubinstein H, Calton GJ. First aid for jellyfish envenomation. South Med J. 1983;76:870-872.

11. Nomura JT, Sato RL, Ahern RM, Snow JL, Kuwaye TT, Yamamoto LG. A randomized paired comparison trial of cutaneous treatments for acute jellyfish (Carybdea alata) stings. Am J Emerg Med. 2002;20:624-626.

12. Burnett JW, Calton GJ, Burnett HW, Mandojana RM. Local and systemic reactions from jellyfish stings. Clin Dermatol. 1987;5:14-28.

13. Yamamoto T, Takiwaki H, Arase S, Ohshima H. Derivation and clinical application of special imaging by means of digital cameras and Image J freeware for quantification 
of erythema and pigmentation. Skin Res. Technol. 2008; $14: 26-34$.

14. Diffey BL, Oliver RJ, Farr PM. A portable instrument for quantifying erythema induced by ultraviolet radiation. $\mathrm{Br} \mathrm{J}$ Dermatol. 1984;111:663-672.

15. Schneider CA, Rasband WS, Eliceiri KW. NIH Image to ImageJ: 25 years of image analysis. Nat Methods. 2012;9:671-675.
16. Sarkar D. Lattice: Multivariate Data Visualization with $R$ New York, NY: Springer; 2008.

17. Moont R, Pud D, Sprecher E, Sharvit G, Yarnistsky D. 'Pain inhibits pain': is pain modulation simply due to distraction? Pain. 2010;150:113-120.

18. Burnett JW. Treatment of Atlantic cnidarian envenomations. Toxicon. 2009;54:1201-1205. 